\section{Underestimation of curvature and task dependence in visual perception of form*}

\author{
VEIJO VIRSU \\ University of Helsinki, Helsinki 17, Finland
}

Perceived curvatures of circular arcs were compared with those of circles. The length and orientation of the arcs varied. In all cases the curvatures of the arcs were underestimated, and the error was a decreasing exponential function of arc length. The results are consistent with the notion that tendency (efferent readiness) to perform rectilinear eye movements produces underestimation of curvature. The results discredited the explanation of the "Gibson normalization effect" as an instance of increase in perceptual accuracy. The overestimation of curvature found in earlier studies was interpreted as resulting from an inappropriate perceptual task and uncontrolled effects of illusions of extent.

Piaget and Vurpillot (1956) reported that the curvature of an arc segment is overestimated if the length of the arc is less than approximately $220 \mathrm{deg}$ of the center angle. The results of Coren and Festinger (1967) confirmed this finding. In both studies the overestimation of curvature was inferred from underestimation of the length of the chord of the arc and overestimation of the width of the arc-segment figure when these dimensions were compared with neutral extents.

However, inferring apparent curvature from data concerned with apparent linear dimensions of arcs may be an invalid procedure. In both studies the intention was to measure the subjective copy of a physical curvature, but because curvature is defined as the inverse of the radius of curvature, curvature alone is not sufficient to determine the height or width of an arc. Therefore, a size feature of the arc is necessarily involved with a height or width estimate, and this size feature may be misperceived independently of curvature.

If the perceptual task is arranged so that no size estimations are involved, as in Fig. 1, the curvature of an arc seems to be underestimated: The two points and the arc are physically located on the circumference of the same circle, but they are not perceived that way, for the are seems to be a part of a larger circle whose interior contains the two points. Underestimation of curvature was also found consistent with the errors that Os made in their estimates of the location of intersection in a figure consisting of two converging arcs (Virsu, 1971). Thus, the estimates of the continuations of ares indicate that the curvatures of short ares are underestimated. In this method. however, it is possible that the "true" apparent curvature is confounded with magined continuations of arcs.

orms an interesting theoretical issue for and Festinger used their result "Gibson normalizat straightening effect is an instance improvement in perceptual accuracy geometrical illusions during repeate estimations. They showed that inspection. However, if the curvature is not initially overestimated but is continuations of arcs, the results of Coren and Festinger require a reinterpretation, and their explanation of the Gibson effect becomes discredited.

The second reason is that overestimation of curvature is at variance with predictions based on eye-movement tendencies. The earlier study (Virsu, 1971) tested the decreased, indeed, with prolong observations concerning the apparent prediction that the tendency to perform rectilinear and horizontal or vertical eye movements (inferred from a review of eye-movement data) can explain some aspects of the perception of geometric forms. Assuming that movement tendencies (efferent readinesses) determine conscious perception of form, at least in part, as theorized by Festinger, Burnham, Ono, and Bamber (1967), underestimation of curvature of short arcs should result from the rectilinear tracking tendency.

The argument is as follows: Every arc activates an implicit tendency to perform rectilinear eye movements, provided that tracking of the arc contour with eye movements can help toward the solution of the perceptual problem given to the $\mathrm{O}$. If the tendency to perform rectilinear eye movements determined perceived curvature completely, every arc would be perceived as a straight line. However, when attempts are made to follow the curvilinear form with explicit rectilinear eye movements, the arc contour disappears from the fovea or from an intended point on the fovea. This produces afferent feedback that induces corrections both to eye movements and to the movement tendency that originated the movements. As a result of the adjustments, perception (determined by the movement tendency) becomes more veridical. The amount of corrective feedback depends upon the width of the arc. Hence, if curvature is held constant, the amount of corrective afferent feedback increases as a function of the arc length. When the arc is very short, the amount of the corrective feedback associated with the arc is negligible and a strong underestimation of curvature occurs. With increasing arc length, the amount of the corrective movements required increases and the underestimation of curvature diminishes accordingly. Therefore, the predicted underestimation of curvature is a monotonically decreasing function of arc length.

Two experiments were conducted as an attempt to resolve misperception of curvature as a function of arc length. It was assumed that estimations of the curvatures of arcs, as compared with curvatures of

\footnotetext{
*Supported by Kamarineuvos H. Rosenberg's travel grant from the University of Helsinki. I am grateful to D.J. Weintraub and D. H. Krantz tor comments, criticism, and the use of their research facilities.
}

Fig. 1. Underestimation of curvature. 
Table 1

Apparent Radii of Curvature (R) and $t$ Values of the Errors in Apparent Curvature

Arc Length (Deg)

\begin{tabular}{|c|c|c|c|c|c|c|c|c|c|c|c|c|}
\hline & 9 & 18 & 36 & 72 & 108 & 144 & 180 & 216 & 252 & 288 & 324 & 342 \\
\hline \multicolumn{13}{|c|}{ Experiment 1} \\
\hline $\mathrm{R} \mathrm{cm}$ & 7.72 & 6.92 & 5.99 & 5.32 & 5.24 & 5.28 & 5.24 & 5.26 & 5.10 & 5.06 & 5.02 & 5.12 \\
\hline$t$ & 10.78 & 8.96 & 6.39 & 1.94 & 1.45 & 4.50 & 6.00 & 3.35 & 2.13 & 1.12 & 0.47 & 1.69 \\
\hline \multicolumn{13}{|c|}{ Experiment 2} \\
\hline $\mathrm{R} \mathrm{cm}$ & 6.92 & 6.51 & 6.04 & 5.32 & 5.36 & 5.17 & 5.21 & 5.17 & 5.15 & 5.24 & 5.09 & 5.08 \\
\hline $\mathrm{t}$ & 8.49 & 6.43 & 5.57 & 2.68 & 3.57 & 2.08 & 4.09 & 5.15 & 4.00 & 5.60 & 1.58 & 1.90 \\
\hline
\end{tabular}

$P(|t| \geqslant 2.09)=.05 ; P(|t| \geqslant 3.88)=.001$

circle contours, could lead to satisfactorily pure estimates of relative apparent curvatures of the arcs. Because this method is based on direct comparisons between two curvatures, the perceptual task should be relevant to the problem of measuring apparent curvature. Two different arc orientations were used. In Experiment 1 the (imagined) chords of the arcs were vertical, and in Experiment 2 they were tilted $45 \mathrm{deg}$. If the tendency-to-movement explanation is correct, a stronger underestimation might occur in the vertical orientation, because the tendency to vertical movements presumably enhances the tendency to perform rectilinear eye movements in that case.

\section{EXPERIMENTS}

\section{Observers}

Twenty students, 15 women and 5 men, from an introductory psychology course at the University of Michigan volunteered as Os.

\section{Stimuli}

Two sets of 12 test arcs, with arc lengths from 9 to $342 \mathrm{deg}$ of the center angle (see Table 1), were drawn on $20 \times 20 \mathrm{~cm}$ pieces of cardboard. In the set used in Experiment 1, the arcs were oriented vertically, the open side to the right, and in the set used in Experiment 2, the arcs were drawn in position where the chords of the arcs, if drawn, would have been tilted $45 \mathrm{deg}$ counterclockwise from the vertical position. The radius of curvature of every arc was $5 \mathrm{~cm}$. The centers of gravity of the arcs were approximately in the middle of the sheets.

Two identical sets of 11 comparison circles were drawn on $20 \times 22 \mathrm{~cm}$ sheets of cardboard. The radii of curvature of the comparison circles were $3.64,3.85,4.08$, $4.35,4.65,5.00,5.40,5.88,6.45,7.14$, and $8.00 \mathrm{~cm}$. Pilot experiments showed that the raw data distributions are symmetrical if the comparison circles are spaced in equal curvature steps and skewed if equal steps are used in the radius of curvature. Therefore, equal steps of curvature $\left(.015 \mathrm{~cm}^{-1}\right)$ were used in spacing the comparison circles. The centers of the comparison circles were placed about $5 \mathrm{~mm}$ above and to the right of the middles of the sheets in order to prevent the use of the edges of the sheets as possible size cues. The longer edges of the sheets were vertical. Line thickness was $.3 \mathrm{~mm}$ in both the arcs and the circles.

\section{Procedure}

A forced-choice version of the method of constant stimuli was used. An arc and a set of the 11 comparison circles were placed on a stand in front of $O$. One comparison circle was visible at a time next to an arc. The $O$ judged whether the circumference of the circle was less or more curved than the arc and indicated his judgment by placing the circle in one of two piles labeled accordingly. He handled the whole set of the 11 comparison circles in this manner. The procedure was performed once with every arc.

All Os participated in both experiments. Every second $O$ started with Experiment 1 and the rest started with Experiment 2. Written instructions with illustrative figures were continuously visible in front of $\mathrm{O}$ to eliminate any confusions, and the concept of curvature was explained to $\mathrm{O}$ before the experiment. The stimulus figure sheets were perpendicular to $O$ 's line of sight and at $60-\mathrm{cm}$ distance from $O$ 's eyes. The presentation order of the 12 arcs in each experiment was determined independently for each $O$ by a table of random numbers, and the order of comparison circles was randomized by shuffling (the two sets of the circles allowed $E$ to shuffle one set while $\mathrm{O}$ was working with the other). The locations of the piles for less and more curved circles, with respect to $\mathrm{O}$, were balanced, but an arc figure was always presented on the left of the comparison circles.

\section{RESULTS}

In both orientations the variances and the number of nontransitive responses were small, with arcs from 144 to $342 \mathrm{deg}$ (11 nontransitive from 280 responses). The PSE for each arc and $O$ combination was "less curved" and "more curved" responses; in the case of the 11 determined as the middle value between nontransitive answers, the PSE was determined as the average of the PSEs obtained. The means and standard deviations were calculated for these PSEs in the usual statistical manner. With arcs from 9 to $108 \mathrm{deg}$, the number of nontransitive answers was progressively higher toward the shorter arcs. Therefore, the overall means and standard deviations for these shorter arcs were determined using the least-squares procedure with averaged 2 scores (see Woodworth \& Schlosberg, 1954, p. 205).

The apparent radii of curvature ( $R$ ) are presented in Table 1. Calculations were performed in curvature units (inverses of the radius) that produced approximately normal distributions of individual PSEs. The values of $t$ show how many standard errors the apparent curvatures deviated from the true curvature, $.20 \mathrm{~cm}^{-1}$. The table shows that the radius of curvature of every arc was overestimated, that is, the curvature of every arc was underestimated as expected. From the values of $t$ it is seen that the perceived curvatures deviate statistically significantly from the physical curvatures in most of the arcs $(\mathrm{df}=19$ in each case). The largest errors were obtained with the shortest arcs.

To illustrate the form of the error and the difference between the two experimental conditions, Fig. 2 presents the error as a fraction of the true curvature. The maximum error is $35.2 \%$ from the physically true curvature, but with increasing arc length, $L$, the error quickly falls to a steady $3 \%$ level. The difference between the results of Experiment 1 (vertical orientation) and Experiment 2 (45-deg tilted orientation) is very small. It is possible that there is a genuine difference between the two conditions, at least with the shortest arcs, but in the present experiment the differences were not large enough to reach statistical significance. The direction of the difference is as expected, because with short arcs more underestimation was produced by the vertical arcs than by the tilted arcs.

From the point of view of the tendency-to-movement explanation, the result in Fig. 2 presents underestimation of curvature as a function of the amount of corrective feedback, because the number of corrective eye movements should increase as a function of arc length, the physical curvature held constant. The effect of the hypothetical afferent feedback is very accurately exponential, as the least-squares curve fitted to the joint results of both experiments shows. 1 It is impossible to track long arcs successfully with rectilinear eye movements, and, therefore, the rapid decrement of the error is not surprising. 


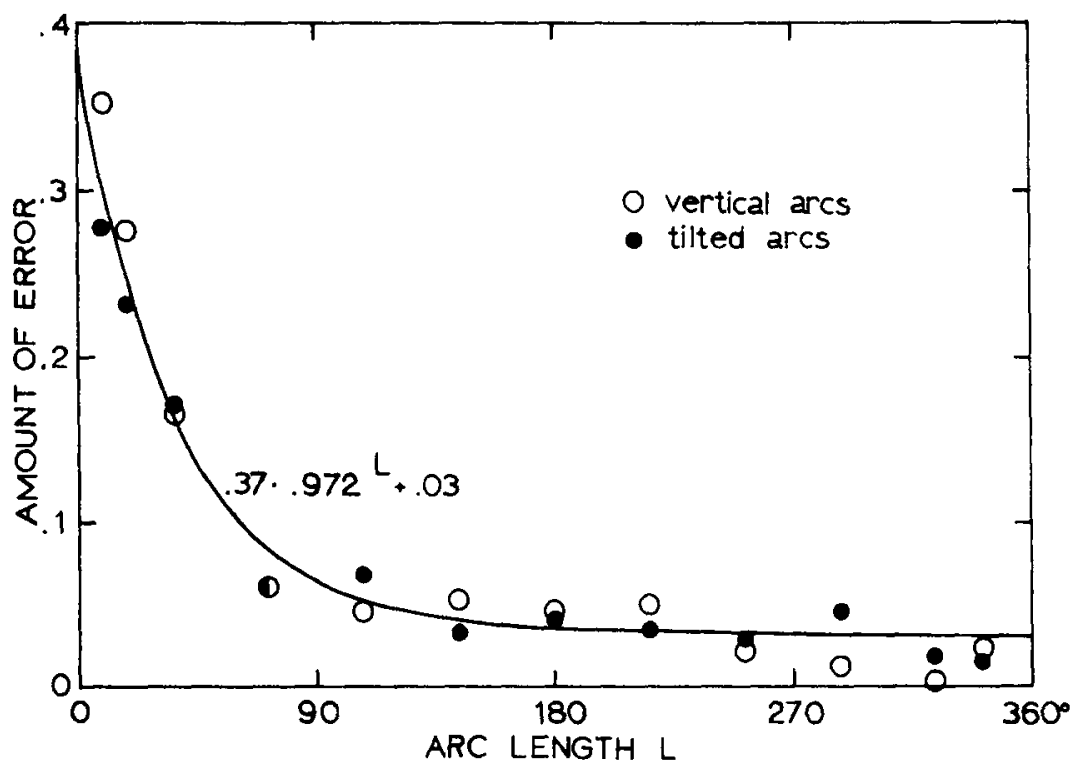

Fig. 2. Errors in perceived curvature as a function of arc length. The amount of error is expressed as a fraction of the true curvature. The formula in the figure refers to the smooth curve fitted to the results.

The error did not vanish with the long arcs, however, for the results indicate a $3 \%$ asymptotical underestimation. This may reflect overestimation of the size of an open figure as compared with a closed figure, for this factor could induce overestimation of the radius of curvature in arcs relative to circles that are closed figures.

The results of the earlier experiment concerning the perceived location of intersection (Virsu, 1971) indicated 17\% underestimation of the curvatures of the 30 -deg arcs and $2 \%$ underestimation of the curvatures of the 90-deg arcs used in the experiment. The corresponding values in the present experiment are $19 \%$ and $6 \%$, interpolated linearly from the error means. These values do not differ essentially from the values found in the intersection experiment. Moreover, if the overestimation of the size of open figures affects the present results, the pure curvature underestimation values could be about $3 \%$ lower, or $16 \%$ and $3 \%$, matching the data of the intersection experiment almost perfectly.

a

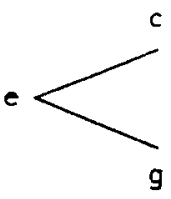

\section{OVERESTIMATION OF CURVATURE: AN INTERPRETATION}

The form of the present error function is such that it cannot be made to agree with the function by Piaget and Vurpillot (1956). The present results show decreasing underestimation of curvature with ares from 9 to $342 \mathrm{deg}$, but the results of Piaget and Vurpillot showed first increasing and then decreasing overestimation of curvature that changed to an increasing underestimation with over 220-deg arcs and diminished when 360 deg was approached.

One possible explanation for the discrepant results of Piaget and Vurpillot (1956) and (oren and Festinger (1967) is that their method confounds illusions of extent with inisperception of curvature. Coren and Festinger paid attention to the possibility that overestimation of the width of an open figure might induce overestimation of the width in the arcs: Yet, both studies ignored the possible effects of the Müller-Lyer illusion. Data by Mountjoy (1966) and Kristof (1964) show that a Müller-Lyer type of illusion occurs

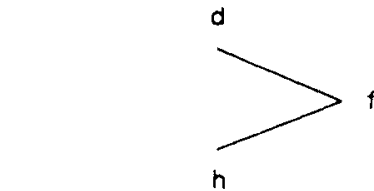

i not only in the conventional form between the vertices of two arrowheads but also between two arrowheads from the outermost tip of one arrowhead oblique to the outermost tip of another arrowhead oblique, or when all the obliques are deleted on one side of the Müller-Lyer figure. When estimating the length of the imagined chord in an arc shorter than $180 \mathrm{deg}$, the length should be underestimated as a version of the Müller-Lyer illusion (ingoing fins). This underestimation of the chord was found in both studies and was interpreted to indicate overestimation of curvature. In addition, the length of the chord was overestimated in the experiments by Piaget and Vurpillot in arcs longer than approximately $220 \mathrm{deg}$ (see also Piaget, 1961 , p. 49), which agrees with the outgoing-fins case of the Müller-Lyer. Because the error in Mountjoy's variant of the Müller-Lyer illusion diminishes with prolonged inspection (Mountjoy, 1966), the decrement effect found by Coren and Festinger may be a sole effect of the Muller-Lyer illusion that is irrelevant to apparent curvature and to the Gibson effect. The overestimation of the width of an arc may be a compensatory expansion resulting from the underestimation of the height of the arc, for this kind of compensation would be necessary for maintaining apparent evenness of the curvature of the arc with the height distortion.

It is also possible that Piaget and Vurpillot and Coren and Festinger did not measure curvature at all, but only illusions of extent. Figure 3 is somewhat complicated but it illustrates the point. Distances $\mathrm{cd}$, gh, and ij (excluding the points) are equal, but ij looks shorter than the two other distances as a case of the Müller-Lyer illusion (cf. Mountjoy, 1966; Kristof, 1964). Distances ef and $a b$ (including the points) are also equal, but ab looks longer, as in the standard Müller-Lyer illusion. Line Segments ec and fd are physically directed toward Point a and Line Segments eg and fh toward i. In addition, Line Segment hf is pointing to $b$. As examples of tilt illusions discussed by Weintraub and Virsu (1971), the lines, if continued rectilinearly, do not seem to go through the points: Points $a$ and $b$ seem to be too high and Point $i$ too low to be on the continuations of the respective line segments. These two sets of misperceptions, those of extent and direction, are incompatible: to satisfy the geometry of the Müller-Lyer illusion, the lines should be tilted or displaced in a manner that is in contradiction to the alignment illusions. For instance, Line Segment hif should be tilted

Fig. 3. Contradiction of illusions of extent and direction. 
counterclockwise to make it aligned with Points $i$ and $b$, but it should be tilted clockwise in order to get Distance Pairs gh and $\mathrm{ij}$, and ef and $a b$ perceptually equal. ${ }^{2}$

Figure 3 shows that illusions of length and direction can take place independently of each other, for the same stimulus display produces contradictory misperceptions, depending on the comparison task assumed by an $\mathrm{O}$. It would be erroneous to infer apparent directions from apparent extents and vice versa. It is possible that some basic attributes, such as length and direction of visual patterns, are processed sequentially and independently of each other in the visual system. ${ }^{3}$ If curvature belongs to these basic attributes, which is plausible on the basis of the present results, the task to estimate the height and width of an arc does not yield any information about apparent curvature, since the task is perceptually irrelevant to curvature estimation.

\section{CONCLUSIONS}

Different methods lead to different results concerning apparent curvature. Estimates of the linear dimensions of arcs suggest overestimation of the curvature of short arcs. However, two methods based on the curvilinear properties of arcs, the estimation of the continuations of arcs and the comparisons of the apparent curvature of arcs with the curvatures of circle contours, both indicate underestimation of the curvature of short arcs.

As far as it is meaningful to refer to apparent curvature without a reference to the method with which it is measured, the curvature of a single arc seems to be underestimated, for: (1) the two methods based on the curvilinear properties of arcs yielded consistent results; (2) if the absolute curvatures of the shortest arcs in the present experiment had been actually overestimated, an implausible amount of overestimation of curvature should be assumed for circle contours; (3) the perceptual task of the present experiment was directly relevant to curvature estimation; and (4) the earlier results can be understood on the basis of the Müller-Lyer illusion, but the present results cannot be reduced to any known illusion.

The results make questionable the explanation by Coren and Festinger that the "Gibson normalization effect" is based on an initial overestimation of curvature and its decrement with prolonged inspection. The results are consistent with the assumption that the tendency to perform rectilinear eye movements produces underestimation of curvature.

\section{REFERENCES}

CHIANG, C. A new theory to explain geometrical illusions produced by crossing lines. Perception \& Psychophysics, 1968, 3, 174-176.

COREN, S., \& FESTINGER, L. An alternative view of the "Gibson normalization effect." Perception \& Psychophysics, 1967, 2, 621.626.

FESTINGER, L., BURNHAM, C. A., ONO, H., \& BAMBER, D. Efference and the conscious experience of perception. Journal of Experimental Psychology Monograph, 1967. 74 (Whole No. 637).

GIBSON, J. J. Adaptation, aftereffect, and contrast in the perception of curved lines. Journal of Experimental Psychology, 1933, $16,1-31$.

HABER, R. N. Nature of the effect of set on perception. Psychological Revicw, 1966, 73, 335-351.

KRISTOF, W. Eine quantitative Analyse der Müller-Lyer Täuschung. Zeitschrift für Psychologie, 1964, 169, 84-97.

MOUNTJOY, P. T. New illusory effect of the Müller-Lyer figure. Journal of Experimental Psychology, 1966.71, 119-123.

PIAGET, J. Les mécanismes perceptifs. Paris: Presses Universitaires de France, 1961.

PIAGET, J., \& VURPILLOT, E. La surestimation de la courbure des arcs de cercle. Archives de Psychologie, 1956, 35.215-232.
VIRSU, V. Tendencies to eye movement, and misperception of curvature, direction, and length. Perception \& Psychophysics, 1971, 9, 65-73.

WEINTRAUB, D. J., \& VIRSU, V. The misperception of angles: Estimating the vertex of converging line segments. Perception \& Psychophysics, 1971, 9, 5-8.

WOODWORTH, R. S., \& SCHLOSBERG, H. Experimental psychology. London: Methuen, 1954.

\section{NOTES}

1. To be accurate, the amount of corrective movements is probably related directly to the maximum deviation of the arc contour from the rectilinear path that would be followed if the arc were tracked in agreement with the tendency to perform rectilinear eye movements. This maximum is the same as the width of the arc. Therefore, the argument $L$ of the exponential function should be replaced by $r(1-\cos 1 / 2 L)$, where $t$ is the radius of curvature of the arc. This correction does not, however, affect significantly the form of the function in Fig. 2, and the corrected function would yield as good a fit to the data as the uncorrected one does.

2. The apparent tilts of Line Segments hf and fd are in contradiction to Chiang's (1968) explanation of the geometrical illusions that involve intersecting lines. According to his explanation, the retinal image of an intersection is blurred, which produces a perceptual displacement of the intersection. If this were true, in perception $\mathrm{hf}$ should be inclined counterclockwise, but it is inclined clockwise, and fd should be inclined clockwise, but it is inclined counterclock wise.

3. The task dependence of perception in Fig. 3 supports the perceptual-tuning hypothesis of selective attention (Haber, 1966) as contrasted to selection in memory processes, for no memory processes should be involved in the present demonstration. When an $O$ directs his attention to the directions of the lines, he does not perceive the distances between the lines and vice versa-otherwise the incompatibility of the perceptual outcomes is difficult to understand. The selection is not based on eye movements, for both the length and tilt illusions can occur in spite of fixating the same point, e.g., Point h, given a sufficiently small visual angle.

(Accepted for publication August 4, 1970.) 